\title{
Origins of the Instability of Non-precious HER Catalysts at
}

\section{Open Circuit Potential}

\author{
Zhenbin Wang, ${ }^{1, \dagger}$ Ya-Rong Zheng, ${ }^{1,3, \uparrow}$ Joseph Montoya, ${ }^{2}$ Degenhart Hochfilzer, ${ }^{1}$ Ang Cao, ${ }^{1}$ \\ Jakob Kibsgaard, ${ }^{1}$ Ib Chorkendorff, ${ }^{1}$ and Jens K. Nørskov ${ }^{1, *}$
}

${ }^{1}$ Department of Physics, Technical University of Denmark, 2800 Kongens Lyngby, Denmark

${ }^{2}$ Toyota Research Institute, Los Altos, CA 94022, USA

${ }^{3}$ Anhui Province Key Laboratory of Advanced Catalytic Materials and Reaction Engineering, School of Chemistry and Chemical Engineering, Hefei University of Technology, 230009, Hefei, China.

†These authors contributed equally

E-mail: jkno@dtu.dk

\begin{abstract}
Non-precious hydrogen evolution reaction (HER) catalysts commonly suffer from severe dissolution under open circuit potential (OCP). In this work, using calculated Pourbaix diagrams, we quantitatively analyze the stability of a set of well-known active HER catalysts $\left(\mathrm{MoS}_{2}, \mathrm{MoP}, \mathrm{CoP}, \mathrm{Pt}\right.$ in acid, and $\mathrm{Ni}_{3} \mathrm{Mo}$ in base) under working conditions. We determine that the large thermodynamic driving force towards decomposition created by the electrode/electrolyte interface potential is responsible for the substantial dissolution of non-precious HER catalysts at OCP. Our analysis further shows the stability of HER catalysts in acidic solution is ordered as Pt $\sim \mathrm{MoS}_{2}>\mathrm{MoP}>\mathrm{CoP}$, which is confirmed by the measured dissolution rates using an inductively
\end{abstract}


coupled plasma mass spectrometer. Based on gained insights, we suggest strategies to circumvent the catalyst dissolution in aqueous solution.

Electrochemical water splitting utilizing renewable energy sources enables sustainable hydrogen production. The development of active and stable non-precious electrocatalysts for hydrogen evolution reaction (HER) would make the deployment of water electrolyzers more attractive. Over the past decades a large volume of research from both experiment and computation has been devoted to the design and search for active non-precious HER catalysts that can achieve reaction rates comparable to Pt. ${ }^{1-13}$ However, studies pertaining to catalyst stability under operating conditions lag far behind. ${ }^{14,15}$ Accurately characterizing the stability of an HER catalyst in an experiment is challenging. Similarly, there are few standardized methods in computation to estimate stability efficiently and accurately. More importantly, non-precious HER catalysts commonly suffer from severe corrosion at open circuit potential (OCP), ${ }^{16-18}$ which is a well-known problem for Ni-based HER catalysts in alkaline media, ${ }^{19-21}$ but has been largely overlooked in the development of non-precious HER catalysts in acid solutions. Using an inductively coupled plasma mass spectrometer (ICP-MS), Ledendecker et al. ${ }^{18}$ recently investigated the stability of binary metallic compounds $\left(\mathrm{WC}, \mathrm{MoS}_{2}, \mathrm{Ni}_{5} \mathrm{P}_{4}\right.$ and $\left.\mathrm{Co}_{2} \mathrm{P}\right)$ and their corresponding metals under HER conditions in acid and found that the dissolution rate of these catalysts at OCP is much higher than during electrocatalysis. Nevertheless, the fundamental reason why non-precious HER catalysts experience severe dissolution at OCP remains elusive.

By mapping the chemical potential of species as a function of electrode potential and ambient $\mathrm{pH}$, the Pourbaix diagram serves as a valuable tool in evaluating the stability of an electrocatalyst in aqueous environment. Experimental Pourbaix diagrams sometimes have limited 
predictive capability for a material's stability due to inadequate thermodynamic data. The emergence of large computational materials databases (e.g., the Materials Project ${ }^{22}$ ) created from density functional theory (DFT) calculations provides a convenient and comprehensive way of assessing materials' stabilities in a high-throughput fashion. ${ }^{23}$ Central to the applicability of these computational methods is that they are properly benchmarked against experiment. It is for instance found that Pourbaix diagrams calculated using the popular Perdew-Burke-Ernzerhof (PBE) functiona ${ }^{24}$ can be quite inaccurate in predicting aqueous stability in certain cases. ${ }^{25}$ Leveraging the high accuracy of the strongly constrained and appropriately normed (SCAN) functional ${ }^{26}$, we recently developed a simple method to accurately calculate the Pourbaix diagram of a given oxide. ${ }^{25}$ In this work, we use this as a basis for analyzing the stability of four well-known active non-precious HER catalysts $\left(\mathrm{MoS}_{2}, \mathrm{MoP}\right.$ and $\mathrm{CoP}$ in acid, and $\mathrm{Ni}_{3} \mathrm{Mo}$ in base) under working conditions and determine origins of the instability at OCP. The predicted stability of these catalysts is further verified by the measured dissolution rate using ICP-MS. In light of obtained insights, we finally discuss strategies to circumvent the dissolution of HER catalysts.

$\mathrm{MoS}_{2}$ is a well-known, reasonably active non-precious HER catalyst. ${ }^{1,2}$ It has been reported to be stable under acidic HER conditions, but dissolves at $\mathrm{OCP}$ in experiment. ${ }^{17,18} \mathrm{Fig}$. 1A presents the SCAN-calculated Mo-S Pourbaix diagram. Unless stated otherwise, a default aqueous ion concentration of $10^{-6} \mathrm{M}$ was used in the Pourbaix diagram generation. The color bar in the diagram shows the scale of aqueous stability of $\mathrm{MoS}_{2}$. In DFT calculations, the aqueous stability of a material is quantitatively estimated by the calculated Pourbaix decomposition free energy $\left(\Delta G_{\mathrm{pbx}}\right){ }^{27}$ which is defined by the chemical potential difference between the material of interest (e.g., $\mathrm{MoS}_{2}$ ) and the stable chemical species, including any reaction-balancing electrons, protons, or water molecules at the corresponding electrode potential and $\mathrm{pH}$ on the diagram. The reaction 
that defines $\Delta \mathrm{G}_{\mathrm{pbx}}$ may be written as $[$ Reactants $]+\mathrm{aH}_{2} \mathrm{O} \rightarrow[$ Products $]+\mathrm{mH}^{+}+n e^{-}$, where a, $\mathrm{m}$, and $\mathrm{n}$ are determined by balancing the oxygen, hydrogen, and charge with the ambient water, proton, and electron species, whose chemical potential is determined by the $\mathrm{pH}$ and potential. By definition, a material with $\Delta G_{\mathrm{pbx}}=0$ is the most stable and will appear on the Pourbaix diagram. The larger $\Delta G_{\mathrm{pbx}}$, the more unstable of a material in aqueous solution. As shown in Fig. $1 \mathrm{~A}, \mathrm{MoS}_{2}$ is predicted to be stable in acid around zero potential (0 vs. RHE). Fig. 1B shows the calculated $\Delta G_{\mathrm{pbx}}$ as a function of potential from $-0.6 \mathrm{~V}$ to $0.5 \mathrm{~V}$ at $\mathrm{pH}=1$. It can be clearly seen that $\mathrm{MoS}_{2}$ is very stable at typical HER operating potentials (e.g., $-0.4 \sim 0 \mathrm{~V})$. At very negative potentials $(<-$ $0.46 \mathrm{~V}), \mathrm{MoS}_{2}$ tends to decompose to $\mathrm{H}_{2} \mathrm{~S}(\mathrm{~s})$ and $\mathrm{Mo}(\mathrm{s})$ with a calculated $\Delta G_{\mathrm{pbx}}$ of $0.2 \mathrm{eV} /$ atom at $-0.6 \mathrm{~V}$. In the literature, it is suggested that a material with $\Delta G_{\mathrm{pbx}}<0.5 \mathrm{eV} /$ atom could be stabilized by self-passivation. ${ }^{27}$ Specifically, the decomposition of $\mathrm{MoS}_{2}$ into more stable solid phases, such as $\mathrm{H}_{2} \mathrm{~S}(\mathrm{~s})$ and $\mathrm{Mo}(\mathrm{s})$ at very negative potentials, requires phase transitions which are generally slow at room temperature. $\mathrm{MoS}_{2}$ is therefore considered to be stable at very negative potentials as well. At high positive potentials $(\mathrm{V}>0.35 \mathrm{~V})$, the calculated $\Delta G_{\mathrm{pbx}}$ steeply increases with increasing potential and arrives at $0.75 \mathrm{eV} /$ atom at $0.5 \mathrm{~V}$, accompanied by the decomposition of $\mathrm{MoS}_{2}$ to $\mathrm{HSO}_{4}{ }^{-}$and $\mathrm{MoO}_{2}(\mathrm{~s})$. This thermodynamic trend explains why $\mathrm{MoS}_{2}$ dissolves significantly at OCP, as experiments show that a positive potential $\left(c a .0 .5 \mathrm{~V}\right.$ in $\left.0.1 \mathrm{M} \mathrm{HClO}_{4}\right)$ is created when bringing $\mathrm{MoS}_{2}$ in contact with the electrolyte. ${ }^{17,18}$ More specifically, the large thermodynamic decomposition driving force induced by the interfacial (electrode/electrolyte) potential is responsible for the instability of $\mathrm{MoS}_{2}$ at OCP. We note that the stability of $\mathrm{MoS}_{2}$ under HER conditions predicted by the Materials Project PBE-calculated (abbr. MP-PBE) diagram is inconsistent with experiment. (Fig. S1) 
A

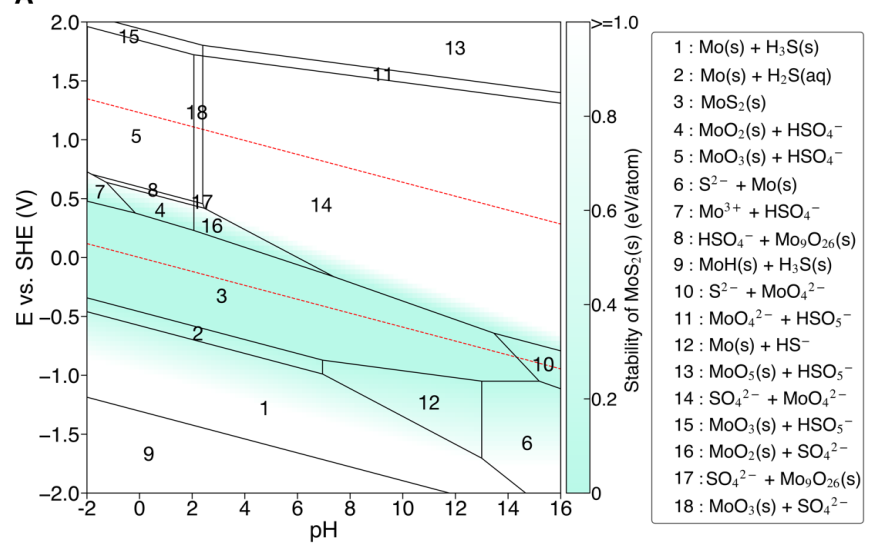

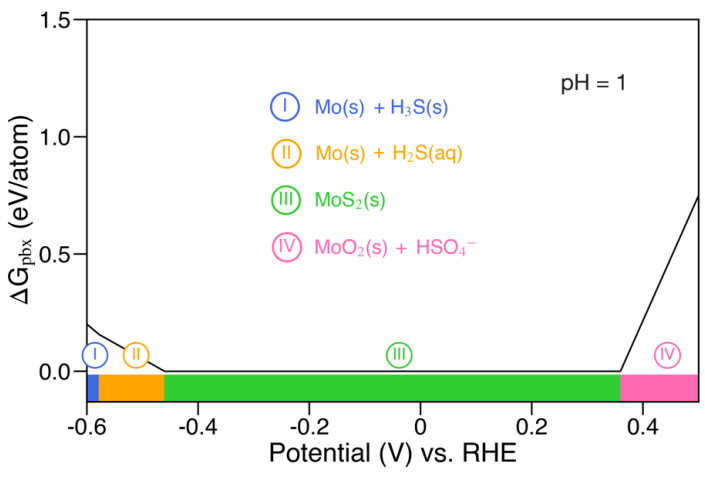

Fig. 1. Stability of $\mathrm{MoS}_{2}$. (A) SCAN-calculated Mo-S Pourbaix diagram generated with aqueous ion concentrations $10^{-6} \mathrm{M}$ at $25^{\circ} \mathrm{C}$. The Lake blue color gauges the stability of $\mathrm{MoS}_{2}$ at relevant potential and $\mathrm{pH}$. The water stability window is shown in red dashed line. (B) Calculated Pourbaix decomposition free energy $\left(\Delta G_{\mathrm{pbx}}\right)$ of $\mathrm{MoS}_{2}$ from the potential $-0.6-0.5 \mathrm{~V} v s$. RHE at $\mathrm{pH}=1$. The projection of $\Delta G_{\mathrm{pbx}}$ onto the potential axis highlights the stable species at the corresponding regions. Roman numerals are only to index the relevant decomposition products.

Prior reports ${ }^{23,25}$ have shown computational Pourbaix diagrams are primarily dictated by calculated formation enthalpies of a given chemistry. Herein, we calculated the formation enthalpies of 83 binary sulfides to examine the generality of this improved performance of the SCAN Pourbaix diagram in stability predictions. Fig. 2A shows calculated formation enthalpies $\left(\Delta \mathrm{H}_{\text {calc }}\right)$ of 83 binary sulfides using the SCAN, PBE and MP-PBE functionals. The MP-PBE refers to the PBE $\Delta \mathrm{H}_{\text {calc }}$ with a sulfur correction of $0.66 \mathrm{eV} / \mathrm{S}^{28}$ applied to all sulfides in the MP. The mean absolute error (MAE) of $\Delta \mathrm{H}_{\text {calc }}$ for SCAN, PBE and MP-PBE functionals are $0.082,0.184$ and $0.205 \mathrm{eV} /$ atom, respectively. The root-mean-square error (RMSE) of $\Delta \mathrm{H}_{\text {calc }}$ for SCAN, PBE and MPPBE functionals are $0.108,0.226$ and $0.245 \mathrm{eV} /$ atom, respectively. Both the MAE and RMSE of the SCAN functional are less than half of those PBE and MP-PBE results, suggesting that the SCAN Pourbaix diagram may have higher accuracy than the MP-PBE for sulfides. Interestingly, we note that the sulfur correction used by the MP to calibrate the error in calculated formation energy for sulfides still results in a significant systematic error. Fig. $2 \mathrm{~B}$ presents the error distribution in $\Delta \mathrm{H}_{\text {calc }}$ of SCAN, PBE and MP-PBE functionals for 83 binary sulfides. 
Compared to experimental values, the SCAN functional yields the smallest deviations, while the PBE and MP-PBE functionals largely overestimate and underestimate the results, respectively.

A

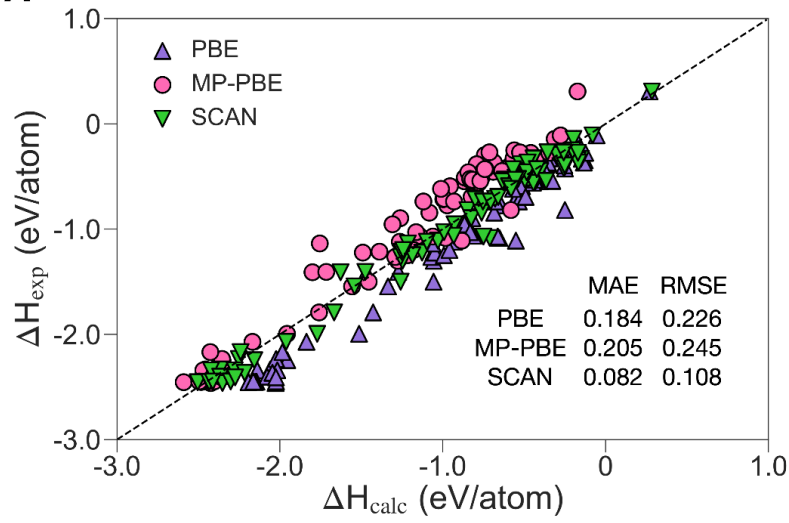

B

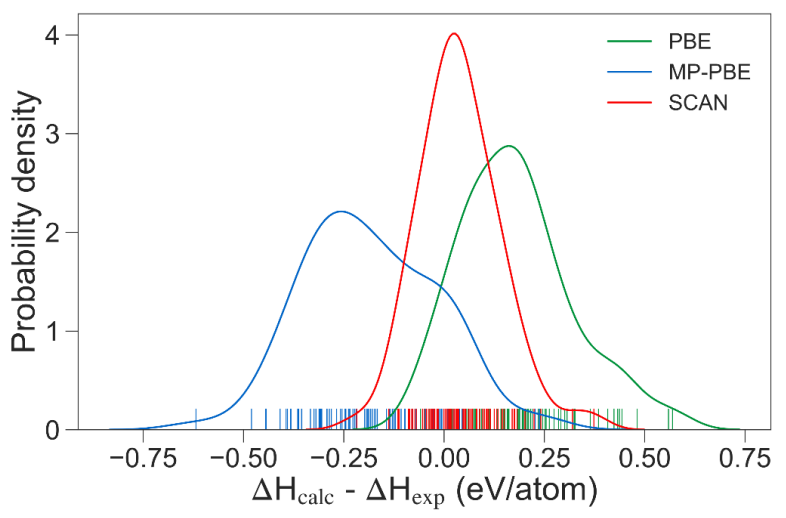

Fig. 2. Formation enthalpies $(\Delta \mathbf{H})$ of sulfides. (A) Experimental $\Delta H_{\text {exp }}$ as a function of calculated $\Delta H_{\text {calc }}$ for 83 binary sulfides. MP-PBE refers to $\Delta \mathrm{H}_{\text {calc }}$ with a sulfur correction of $0.66 \mathrm{eV} / \mathrm{S}^{28}$ adopted by the Materials Project. MAE: mean absolute error; RMSE: root mean squared error (b) Probability density distribution of energy difference between $\Delta \mathrm{H}_{\text {calc }}$ and $\Delta \mathrm{H}_{\text {exp }}$ for 83 binary sulfides. MP-PBE/PBE and experimental data were obtained from the Materials Project $^{22,29,30}$ and Ref ${ }^{31,32}$, respectively. Raw data are provided in Table S1.

Phosphides are another class of materials that are widely studied in search of active nonprecious HER catalysts. ${ }^{33,34}$ Here we selected $\mathrm{MoP}^{7,9}$ and $\mathrm{CoP}^{8,10}$, two active non-precious HER catalysts as representative to investigate their stability behaviors under HER working conditions. Fig. 3A-D show the calculated $\Delta G_{\mathrm{pbx}}$ of MoP and CoP from potentials -0.6 to $0.5 \mathrm{~V}$ at $\mathrm{pH}=1$. Given that $3 d$ transition metals are in general unstable in acidic solution, ${ }^{35}$ we also calculated $\Delta G_{\mathrm{pbx}}$ for these two catalysts at higher aqueous ions concentration $\left(10^{-3} \mathrm{M}\right)$ for comparison. The calculated Pourbaix diagrams of the Mo-P and Co-P chemistries are provided in Figure S2-3. At $10^{-6} \mathrm{M}, \mathrm{MoP}$ exhibits a stable range over $-0.4 \sim-0.2 \mathrm{~V}$, while $\mathrm{CoP}$ is predicted to be unstable or metastable. At $10^{-3} \mathrm{M}$, the stable range of $\mathrm{MoP}$ is significantly expanded with a $\Delta G_{\mathrm{pbx}}$ of 0.66 $\mathrm{eV} /$ atom at $0 \mathrm{~V}$. CoP now appears in a narrow stable range at $\sim-0.3 \mathrm{~V}$ with a $\Delta G_{\mathrm{pbx}}$ of $0.97 \mathrm{eV} /$ atom at $0 \mathrm{~V}$. At very negative potentials $(<-0.5 \mathrm{~V})$, the calculated $\Delta G_{\mathrm{pbx}}$ slightly increases with the formation of $\mathrm{Mo}(\mathrm{s})+\mathrm{PH}_{3}(\mathrm{aq})$ for $\mathrm{MoP}$ and $\mathrm{CoH}(\mathrm{s})+\mathrm{PH}_{3}(\mathrm{aq})$ for $\mathrm{CoP}$. The solid phases in these 
decomposition products may prevent the corrosion of the catalyst because of self-passivation or a high barrier for solid-solid phase transitions. ${ }^{27}$ While the large $\Delta G_{\mathrm{pbx}}(>1.0 \mathrm{eV} /$ atom $)$ beyond $0 \mathrm{~V}$ suggests that these catalysts would be corroded. The experimentally measured OCPs in acid for MoP and CoP are 0.38 V and 0.11 V vs. RHE, respectively. (See Fig. 4A) Similar to $\mathrm{MoS}_{2}$, the severe dissolution of MoP and CoP at OCPs can be therefore attributed to the large thermodynamic driving force to decompose.

Platinum is one of the best HER catalysts in terms of activity and stability in acid. ${ }^{18,36-38}$ As a benchmark reference, we evaluated the stability behavior of $\mathrm{Pt}$ at $\mathrm{pH}=1$ and potential $-0.6 \sim$ 1.0 V, as shown in Fig. 3E. The calculated Pt Pourbaix diagram is presented in Fig. S4. Clearly, Pt is predicted to be very stable under HER conditions in acidic solution, which is consistent with the experimental observation that the dissolution of $\mathrm{Pt}$ in $0.1 \mathrm{M} \mathrm{HClO}_{4}$ can hardly be detected by ICP-MS. ${ }^{18}$ With our previous discussions, we conclude the stability of HER catalysts under hydrogen evolution is in ordered as $\mathrm{Pt} \sim \mathrm{MoS}_{2}>\mathrm{MoP}>\mathrm{CoP}$. 
A

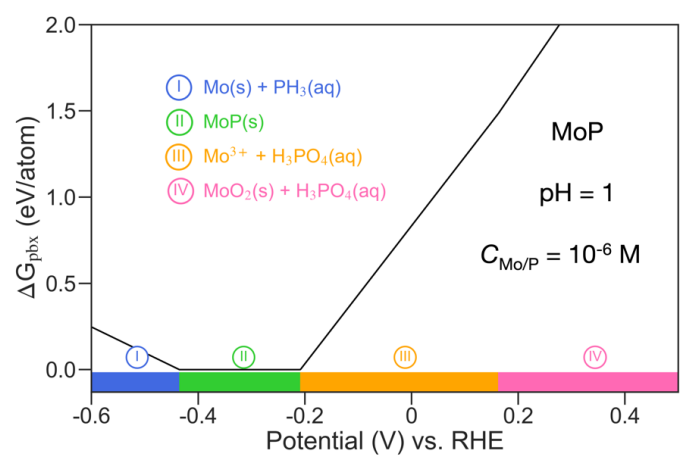

C

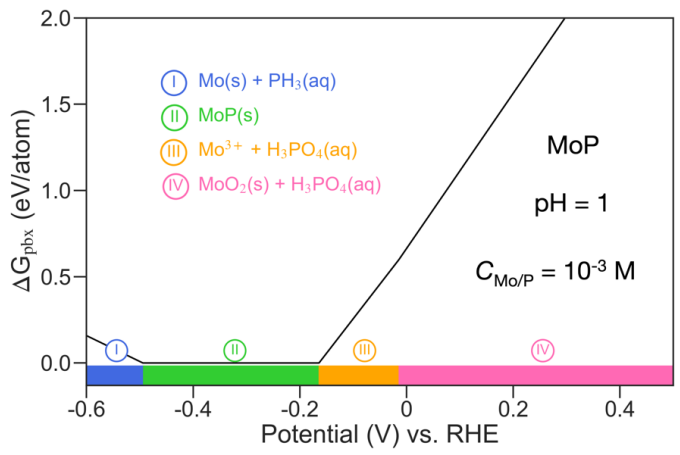

$\mathbf{E}$

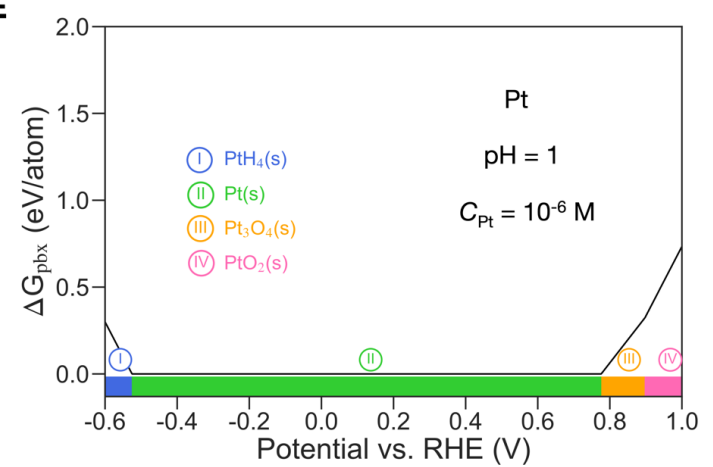

B

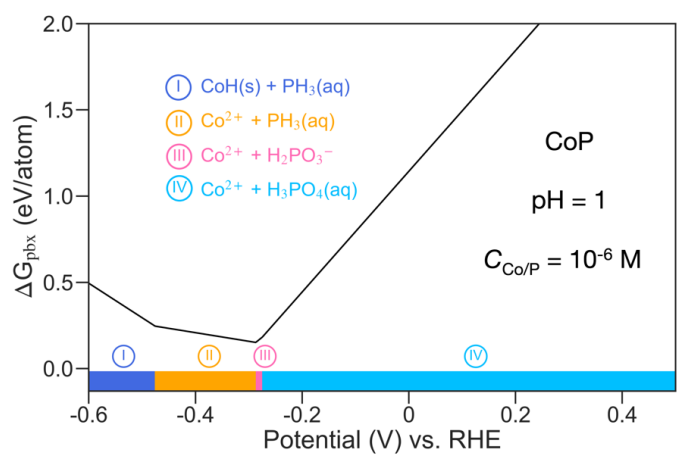

D

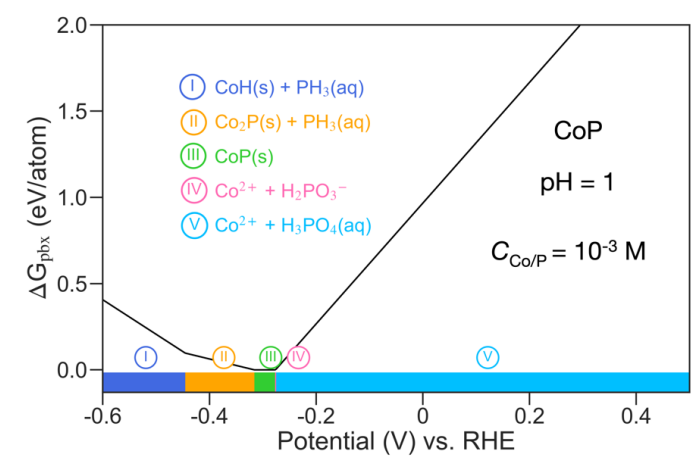

$\mathbf{F}$

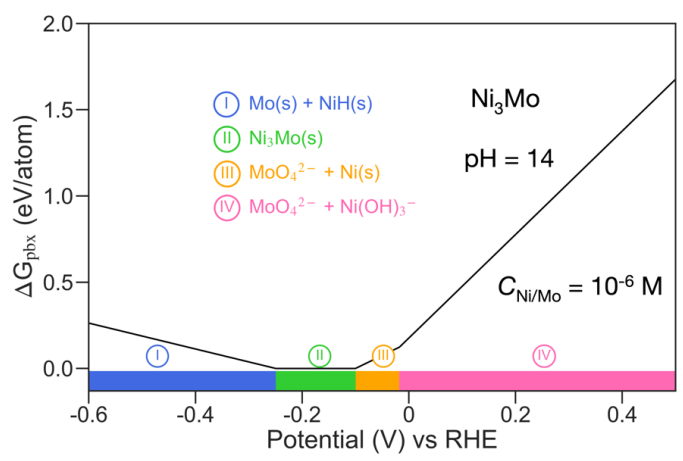

Fig. 3. Stability analysis of typical HER catalysts in both acidic and basic media. Calculated Pourbaix decomposition free energy $\left(\Delta G_{\mathrm{pbx}}\right)$ of $(\mathbf{A}, \mathbf{C}) \mathrm{MoP}$ and $(\mathbf{B}, \mathbf{D}) \mathrm{CoP}$ and $(\mathbf{E}) \mathrm{Pt}$ with different aqueous ion concentrations in acid $(\mathrm{pH}=1)$. (F) Calculated $\Delta G_{\mathrm{pbx}}$ of $\mathrm{Ni}_{3}$ Mo with aqueous ion concentrations of $10^{-6} \mathrm{M}$ in alkaline $(\mathrm{pH}=14)$. The projection of $\Delta G_{\mathrm{pbx}}$ onto the potential axis highlights the stable species at the corresponding regions. Roman numerals are only to index the relevant decomposition products.

In analogy to our analysis of sulfides, we examine the accuracy of the SCAN functional in calculated formation enthalpies for 28 binary phosphides to estimate its performance in Pourbaix diagram calculations. We find that the SCAN functional slightly improves the accuracy of the PBE functional. (Fig. S5A) This small improvement for phosphides, compared to the considerable improvement for sulfides, may result from an inherent inaccuracy of the SCAN functional itself 
or the large uncertainty $( \pm 0.2 \mathrm{eV} /$ atom) of experimental data. (Figure S5B, the uncertainties of $\Delta \mathrm{H}_{\mathrm{exp}}$ for the majority (90\%) of sulfides are lower than $\left.0.03 \mathrm{eV} / \mathrm{atom}.\right)$

Ni-Mo alloys are the most active non-precious HER catalysts in alkaline conditions, whereas it has been found in experiment that Mo leaches out not only during electrocatalysis but also at OCP. ${ }^{21,39-41}$ Based on the calculated Ni-Mo Pourbaix diagram (Fig. S6), we plotted $\Delta G_{\mathrm{pbx}}$ of $\mathrm{Ni}_{3} \mathrm{Mo}$ from potentials -0.6 to $0.5 \mathrm{~V}$ at $\mathrm{pH}=14$, shown in Fig. 3F. In experiment, the measured overpotentials of Ni-Mo alloys are close to $0 \mathrm{~V}^{21,39-42}$, and the measured OCP in $1 \mathrm{M} \mathrm{NaOH}$ is $\sim 0.2 \mathrm{~V}^{43}$. From Fig. 3F, we may observe that $\mathrm{Ni}_{3} \mathrm{Mo}$ decomposes to $\mathrm{MoO}_{4}{ }^{2-}+\mathrm{Ni}(\mathrm{s})$ at potential of $-0.1 \sim 0 \mathrm{~V}$ and $\mathrm{MoO}_{4}{ }^{2-}+\mathrm{Ni}(\mathrm{OH})_{3}{ }^{-}$at potential $>0 \mathrm{~V}$. Similar results are obtained at a higher ion concentration $\left(10^{-4} \mathrm{M}\right)$. (Fig. S7) In particular, the calculated $\Delta G_{\mathrm{pbx}}$ of $\mathrm{Ni}_{3} \mathrm{Mo}$ significantly increases with the potential turning more positive. These results suggest that the leaching of Mo during electrocatalysis and at OCP is likely because of the intrinsic instability of the catalyst and the large thermodynamic decomposition driving force, respectively.

To validate our theoretical predictions, the electrochemical stability of $\mathrm{MoS}_{2}, \mathrm{MoP}$ and $\mathrm{CoP}$ under HER and successive lower working potentials were investigated by chronoamperometry in $\mathrm{H}_{2}$-saturated $0.5 \mathrm{M} \mathrm{H}_{2} \mathrm{SO}_{4}$. Before applying relevant hydrogen evolution potentials, the electrode was brought into the electrolyte under OCP, as shown in Fig. 4A. There is an OCP transition for each catalyst during the first 60 s, which is likely from the catalyst dissolution. The measured OCPs for $\mathrm{MoS}_{2}, \mathrm{MoP}$ and CoP are $0.23 \mathrm{~V}, 0.38 \mathrm{~V}$ and $0.11 \mathrm{~V} v s$. RHE, respectively. After $1 \mathrm{~min}$ at $\mathrm{OCP}$, the potential subsequently stepped down to relevant HER working potentials from 0 to $-0.6 \mathrm{~V}$ vs. RHE (Fig. S9). The electrolyte was then collected after each potential for the further catalyst dissolution analysis by ICP-MS (Fig. S10). Fig. 4B displays the metal dissolution rate at different potentials. All three catalysts show a similar corrosion 
behavior that the dissolution rate at OCP is one order of magnitude higher than that during hydrogen evolution, which is in line with previous study. ${ }^{18}$ Different dissolution rates of these catalysts $\left(\mathrm{MoS}_{2}<\mathrm{MoP}<\mathrm{CoP}\right)$ clearly showcase their distinct stability behaviors in acidic solution, which qualitatively confirms our theoretical predictions. During HER (0 to -0.6 V vs. RHE), each catalyst exhibits a constant dissolution rate at different working potentials. The dissolution ratio of each electrode, (Fig. 4C) computed based on the dissolution rate and total loading of each catalyst, reveals that the main dissolution process occurs at OCPs, and the dissolution ratio of $\mathrm{MoS}_{2}$ is three orders of magnitude higher than that of CoP and MoP. In addition, the stability number (S-number) has been reported to be a good metric to evaluate the stability of an electrocatalyst. ${ }^{44}$ The S-number is defined as the ratio between the amount of generated product and dissolved active center. The average S-numbers of CoP, MoP, and $\mathrm{MoS}_{2}$ were obtained according to the produced $\mathrm{H}_{2}$ (calculated from $\mathrm{Q}_{\text {total }}$ ) and the dissolved Co/Mo (extracted from ICP-MS) during HER (-0.2 to -0.5 V vs. RHE), as shown in Fig. 4D. MoS 2 shows the highest S-number, which is three orders and one order of magnitude higher than those of CoP and MoP, respectively. Note that S-numbers of these catalysts depend on working potentials (Fig. S11) because of the constant dissolution rates of these catalysts during hydrogen evolution. A highperformance HER catalyst is, therefore, expected to possess a higher S-number even at low overpotentials. 

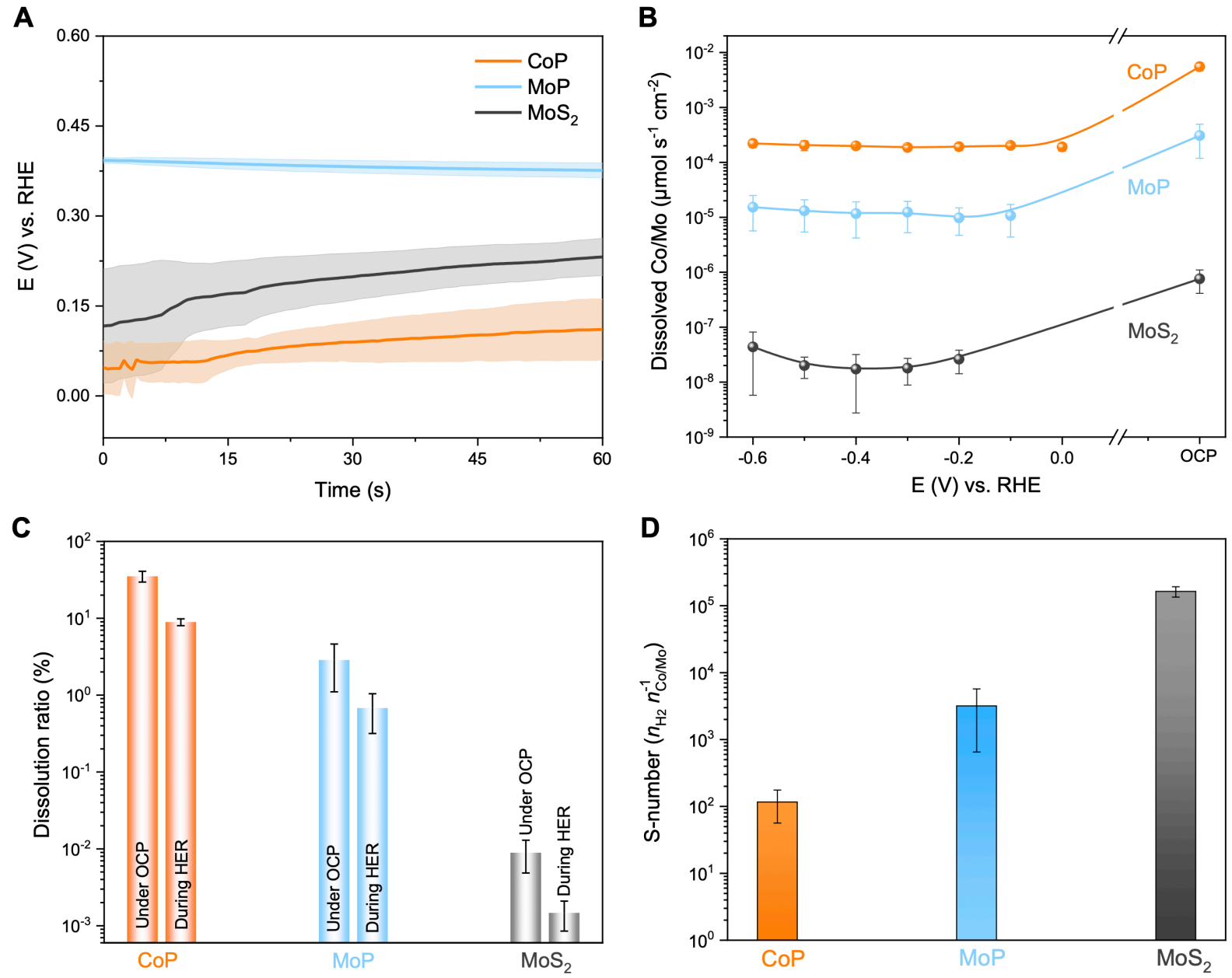

Fig. 4. Corrosion measurements of $\mathrm{MoS}_{2}$, MoP, and CoP during HER process. (A) Open circuit potentials at CoP, $\mathrm{MoP}$, and $\mathrm{MoS}_{2}$ electrodes as a function of time in $\mathrm{H}_{2}$-saturated $0.5 \mathrm{M} \mathrm{H}_{2} \mathrm{SO}_{4}$. (B) Dissolution rates of $\mathrm{CoP}$, MoP, and $\mathrm{MoS}_{2}$ at different potentials. (C) Dissolution ratios of CoP, MoP, and $\mathrm{MoS}_{2}$ at OCP and HER working potentials (0 to $-0.6 \mathrm{~V}$ vs. RHE). (D) The average S-numbers of CoP, MoP, and $\mathrm{MoS}_{2}$ during HER (-0.1 to -0.5 vs. RHE). Average and standard deviations are based on three independent measurements.

We note that the measured OCP for $\mathrm{MoS}_{2}$ in $0.5 \mathrm{M} \mathrm{H}_{2} \mathrm{SO}_{4}(0.23 \mathrm{~V} v s$. RHE) is lower than that in $0.1 \mathrm{M} \mathrm{HClO}_{4}(c a .0 .5 \mathrm{~V})^{18}$. The SCAN Pourbaix diagram predicts $\mathrm{MoS}_{2}$ to be stable up to $0.35 \mathrm{~V}$ (Fig. 1B), which is inconsistent with the experimental results in Fig. 4. While this difference is small $(0.12 \mathrm{~V})$ and does not change our conclusion of $\mathrm{MoS}_{2}$ 's robustness under acidic HER, the discrepancy suggests that there is still some error in either the accuracy of the oxidative corrosion threshold for $\mathrm{MoS}_{2}$ predicted by SCAN or in the comparative interpretation of our results with experiment. 
Based on the above stability analysis of HER catalysts, we propose possible strategies to circumvent the dissolution under working conditions. Since the corrosion of an active HER catalyst during reaction primarily results from the intrinsic instability of the material, the introduction of aqueous-stable species ${ }^{45}$ or coating with aqueous-stable materials ${ }^{46}$ might be conducive to enhancing the catalyst stability. At OCPs, most of, if not all non-precious HER catalysts experience higher dissolution because of the large thermodynamic decomposition driving force. One approach is to develop new active non-precious HER catalysts with a large stability window and minimal OCP. Another, and much easier, approach is to mitigate OCP by applying potential control and never let the catalyst go to $\mathrm{OCP} .{ }^{47}$

We also note the Pourbaix diagram construction is grounded on thermodynamics. Kinetics could also contribute to the stabilization and/or dissolution of an electrocatalyst in aqueous solution. Our results indicate the stability behavior of an electrocatalyst under working conditions could be assessed by simple thermodynamic Pourbaix analysis. Along with the widely used HER activity descriptor, ${ }^{48}$ we anticipate that the protocol of stability analysis demonstrated in this work could contribute to the development of stable and active non-precious electrocatalysts for practical applications.

\section{Supporting Information}

Computational and experimental methods; MP-PBE Mo-S Pourbaix diagram; Mo-P, Co-P, Pt and Ni-Mo Pourbaix diagrams; Formation enthalpies of phosphides; X-ray powder diffraction; Stability measurement protocol; Calculated and experimental formation enthalpies for sulfides and phosphides; Calculated and experimental formation Gibbs free energy of solids and aqueous ions. 


\section{Acknowledgments:}

This work was supported by Toyota Research Institute. Y.-R Zheng acknowledges Dr. Mattia Saccoccio and Dr. Vahid Sharavan at SurfCat for providing MoP samples.

Notes: The authors declare no competing financial interest.

\section{Reference:}

(1) Hinnemann, B.; Moses, P. G.; Bonde, J.; Jørgensen, K. P.; Nielsen, J. H.; Horch, S.; Chorkendorff, I.; Nørskov, J. K. Biomimetic Hydrogen Evolution: $\mathrm{MoS}_{2}$ Nanoparticles as Catalyst for Hydrogen Evolution. J. Am. Chem. Soc. 2005, 127 (15), 5308-5309. https://doi.org/10.1021/ja0504690.

(2) Jaramillo, T. F.; Jørgensen, K. P.; Bonde, J.; Nielsen, J. H.; Horch, S.; Chorkendorff, I. Identification of Active Edge Sites for Electrochemical $\mathrm{H}_{2}$ Evolution from $\mathrm{MoS}_{2}$ Nanocatalysts. $\quad$ Science $\quad$ (80). $\quad$ 2007, $317 \quad$ (5834), 100-102. https://doi.org/10.1126/science.1141483.

(3) Bonde, J.; Moses, P. G.; Jaramillo, T. F.; Nørskov, J. K.; Chorkendorff, I. Hydrogen Evolution on Nano-Particulate Transition Metal Sulfides. Faraday Discuss. 2008, 140, 219-231. https://doi.org/10.1039/b803857k.

(4) Cheng, L.; Huang, W.; Gong, Q.; Liu, C.; Liu, Z.; Li, Y.; Dai, H. Ultrathin WS 2 Nanoflakes as a High-Performance Electrocatalyst for the Hydrogen Evolution Reaction. Angew. Chemie Int. Ed. 2014, 53 (30), 7860-7863. https://doi.org/10.1002/anie.201402315. 
(5) Wan, C.; Regmi, Y. N.; Leonard, B. M. Multiple Phases of Molybdenum Carbide as Electrocatalysts for the Hydrogen Evolution Reaction. Angew. Chemie 2014, 126 (25), 6525-6528. https://doi.org/10.1002/ange.201402998.

(6) Saadi, F. H.; Carim, A. I.; Verlage, E.; Hemminger, J. C.; Lewis, N. S.; Soriaga, M. P. CoP as an Acid-Stable Active Electrocatalyst for the Hydrogen-Evolution Reaction: Electrochemical Synthesis, Interfacial Characterization and Performance Evaluation. J. Phys. Chem. C 2014, 118 (50), 29294-29300. https://doi.org/10.1021/jp5054452.

(7) Chen, X.; Wang, D.; Wang, Z.; Zhou, P.; Wu, Z.; Jiang, F. Molybdenum Phosphide: A New Highly Efficient Catalyst for the Electrochemical Hydrogen Evolution Reaction. Chem. Commun. 2014, 50 (79), 11683-11685. https://doi.org/10.1039/c4cc05936k.

(8) Popczun, E. J.; Read, C. G.; Roske, C. W.; Lewis, N. S.; Schaak, R. E. Highly Active Electrocatalysis of the Hydrogen Evolution Reaction by Cobalt Phosphide Nanoparticles. Angew. Chemie 2014, 126 (21), 5531-5534. https://doi.org/10.1002/ange.201402646.

(9) Xiao, P.; Sk, M. A.; Thia, L.; Ge, X.; Lim, R. J.; Wang, J.-Y.; Lim, K. H.; Wang, X. Molybdenum Phosphide as an Efficient Electrocatalyst for the Hydrogen Evolution Reaction. Energy Environ. Sci. 2014, $7 \quad$ (8), 2624-2629. https://doi.org/10.1039/C4EE00957F.

(10) Kibsgaard, J.; Tsai, C.; Chan, K.; Benck, J. D.; Nørskov, J. K.; Abild-Pedersen, F.; Jaramillo, T. F. Designing an Improved Transition Metal Phosphide Catalyst for Hydrogen Evolution Using Experimental and Theoretical Trends. Energy Environ. Sci. 2015, 8 (10), 3022-3029. https://doi.org/10.1039/c5ee02179k.

(11) Wang, X.; Chen, Y.; Zheng, B.; Qi, F.; He, J.; Li, Q.; Li, P.; Zhang, W. Graphene-like WSe 2 
nanosheets for Efficient and Stable Hydrogen Evolution. J. Alloys Compd. 2017, 691, 698704. https://doi.org/10.1016/j.jallcom.2016.08.305.

(12) Li, J.; Liu, P.; Qu, Y.; Liao, T.; Xiang, B. WSe 2 RGO Hybrid Structure: A Stable and Efficient Catalyst for Hydrogen Evolution Reaction. Int. J. Hydrogen Energy 2018, 43 (5), 2601-2609. https://doi.org/10.1016/j.ijhydene.2017.12.160.

(13) Yang, J.; Mohmad, A. R.; Wang, Y.; Fullon, R.; Song, X.; Zhao, F.; Bozkurt, I.; Augustin, M.; Santos, E. J. G.; Shin, H. S.; Zhang, W.; Voiry, D.; Jeong, H. Y.; Chhowalla, M. Ultrahigh-Current-Density Niobium Disulfide Catalysts for Hydrogen Evolution. Nat. Mater. 2019, 18 (12), 1309-1314. https://doi.org/10.1038/s41563-019-0463-8.

(14) Safizadeh, F.; Ghali, E.; Houlachi, G. Electrocatalysis Developments for Hydrogen Evolution Reaction in Alkaline Solutions - A Review. Int. J. Hydrogen Energy 2015, 40 (1), 256-274. https://doi.org/10.1016/j.ijhydene.2014.10.109.

(15) Masa, J.; Andronescu, C.; Schuhmann, W. Electrocatalysis as the Nexus for Sustainable Renewable Energy: The Gordian Knot of Activity, Stability, and Selectivity. Angew. Chemie - Int. Ed. 2020, 59 (36), 15298-15312. https://doi.org/10.1002/anie.202007672.

(16) Harnisch, F.; Sievers, G.; Schröder, U. Tungsten Carbide as Electrocatalyst for the Hydrogen Evolution Reaction in pH Neutral Electrolyte Solutions. Appl. Catal. B Environ. 2009, 89 (3-4), 455-458. https://doi.org/10.1016/j.apcatb.2009.01.003.

(17) Chen, Z.; Cummins, D.; Reinecke, B. N.; Clark, E.; Sunkara, M. K.; Jaramillo, T. F. CoreShell $\mathrm{MoO}_{3}-\mathrm{MoS}_{2}$ Nanowires for Hydrogen Evolution: A Functional Design for Electrocatalytic Materials. Nano Lett. 2011, 11 (10), 4168-4175. https://doi.org/10.1021/n12020476. 
(18) Ledendecker, M.; Mondschein, J. S.; Kasian, O.; Geiger, S.; Göhl, D.; Schalenbach, M.; Zeradjanin, A.; Cherevko, S.; Schaak, R. E.; Mayrhofer, K. Stability and Activity of NonNoble-Metal-Based Catalysts Toward the Hydrogen Evolution Reaction. Angew. Chemie Int. Ed. 2017, 56 (33), 9767-9771. https://doi.org/10.1002/anie.201704021.

(19) Conway, B. E.; Angerstein-Kozlowska, H.; Sattar, M. A.; Tilak, B. V. Study of a Decomposing Hydride Phase at Nickel Cathodes by Measurement of Open-Circuit Potential Decay. J. Electrochem. Soc. 1983, 130 (9), 1825-1836. https://doi.org/10.1149/1.2120106.

(20) Tilak, B. V; Ramamurthy, A. C.; Conway, B. E. High Performance Electrode Materials for the Hydrogen Evolution Reaction from Alkaline Media. Proc. Indian Acad. Sci. - Chem. Sci. 1986, 97 (3), 359-393. https://doi.org/10.1007/BF02849200.

(21) Divisek, J.; Schmitz, H.; Balej, J. Ni and Mo Coatings as Hydrogen Cathodes. J. Appl. Electrochem. 1989, 19 (4), 519-530. https://doi.org/https://doi.org/10.1007/BF02849200.

(22) Jain, A.; Ong, S. P.; Hautier, G.; Chen, W.; Richards, W. D.; Dacek, S.; Cholia, S.; Gunter, D.; Skinner, D.; Ceder, G.; Persson, K. A. Commentary: The Materials Project: A Materials Genome Approach to Accelerating Materials Innovation. APL Mater. 2013, 1 (1), 011002. https://doi.org/10.1063/1.4812323.

(23) Persson, K. A.; Waldwick, B.; Lazic, P.; Ceder, G. Prediction of Solid-Aqueous Equilibria: Scheme to Combine First-Principles Calculations of Solids with Experimental Aqueous States. Phys. Rev. B 2012, 85 (23), 235438. https://doi.org/10.1103/PhysRevB.85.235438.

(24) Perdew, J. P.; Burke, K.; Ernzerhof, M. Generalized Gradient Approximation Made Simple. Phys. Rev. Lett. 1996, 77 (18), 3865-3868. https://doi.org/10.1103/PhysRevLett.77.3865.

(25) Wang, Z.; Guo, X.; Montoya, J.; Nørskov, J. K. Predicting Aqueous Stability of Solid with 
Computed Pourbaix Diagram Using SCAN Functional. npj Comput. Mater. 2020, 6 (1), 160. https://doi.org/10.1038/s41524-020-00430-3.

(26) Sun, J.; Ruzsinszky, A.; Perdew, J. Strongly Constrained and Appropriately Normed Semilocal Density Functional. Phys. Rev. Lett. 2015, 115 (3), 036402. https://doi.org/10.1103/PhysRevLett.115.036402.

(27) Singh, A. K.; Zhou, L.; Shinde, A.; Suram, S. K.; Montoya, J. H.; Winston, D.; Gregoire, J. M.; Persson, K. A. Electrochemical Stability of Metastable Materials. Chem. Mater. 2017, 29 (23), 10159-10167. https://doi.org/10.1021/acs.chemmater.7b03980.

(28) Zhu, Z.; Chu, I.-H.; Deng, Z.; Ong, S. P. Role of $\mathrm{Na}^{+}$Interstitials and Dopants in Enhancing the $\mathrm{Na}^{+}$Conductivity of the Cubic $\mathrm{Na}_{3} \mathrm{PS}_{4}$ Superionic Conductor. Chem. Mater. 2015, 27 (24), 8318-8325. https://doi.org/10.1021/acs.chemmater.5b03656.

(29) Ong, S. P.; Richards, W. D.; Jain, A.; Hautier, G.; Kocher, M.; Cholia, S.; Gunter, D.; Chevrier, V. L.; Persson, K. A.; Ceder, G. Python Materials Genomics (Pymatgen): A Robust, Open-Source Python Library for Materials Analysis. Comput. Mater. Sci. 2013, 68, 314-319. https://doi.org/10.1016/j.commatsci.2012.10.028.

(30) Ong, S. P.; Cholia, S.; Jain, A.; Brafman, M.; Gunter, D.; Ceder, G.; Persson, K. A. The Materials Application Programming Interface (API): A Simple, Flexible and Efficient API for Materials Data Based on REpresentational State Transfer (REST) Principles. Comput. Mater. Sci. 2015, 97, 209-215. https://doi.org/10.1016/j.commatsci.2014.10.037.

O. Kubaschewski; C. B. Alcock; P. J. Spencer. Materials Thermochemistry, 6th ed.; Oxford: Pergamon Press, New York, USA, 1993.

(32) P.J. Linstrom and W.G. Mallard, E. NIST Chemistry WebBook, NIST Standard Reference 
Database Number 69.

(33) Shi, Y.; Zhang, B. Recent Advances in Transition Metal Phosphide Nanomaterials: Synthesis and Applications in Hydrogen Evolution Reaction. Chem. Soc. Rev. 2016, 45 (6), 1529-1541. https://doi.org/10.1039/c5cs00434a.

(34) Callejas, J. F.; Read, C. G.; Roske, C. W.; Lewis, N. S.; Schaak, R. E. Synthesis, Characterization, and Properties of Metal Phosphide Catalysts for the Hydrogen-Evolution Reaction. Chem. Mater. 2016, $28 \quad$ (17), 6017-6044. https://doi.org/10.1021/acs.chemmater.6b02148.

(35) Pourbaix, M. Atlas of Electrochemical Equilibria in Aqueous Solutions., 2nd ed.; National Association of Corrosion Engineers, Houston, USA, 1974.

(36) Trasatti, S. Work Function, Electronegativity, and Electrochemical Behaviour of Metals. III. Electrolytic Hydrogen Evolution in Acid Solutions. J. Electroanal. Chem. 1972, 39 (1), 163-184. https://doi.org/10.1016/S0022-0728(72)80485-6.

(37) Marković, N. M.; Grgur, B. N.; Ross, P. N. Temperature-Dependent Hydrogen Electrochemistry on Platinum Low-Index Single-Crystal Surfaces in Acid Solutions. J. Phys. Chem. B 1997, 101 (27), 5405-5413. https://doi.org/10.1021/jp970930d.

(38) Hansen, J. N.; Prats, H.; Toudahl, K. K.; Mørch Secher, N.; Chan, K.; Kibsgaard, J.; Chorkendorff, I. Is There Anything Better than Pt for HER? ACS Energy Lett. 2021, 6 (4), 1175-1180. https://doi.org/10.1021/acsenergylett.1c00246.

(39) Lasia, A. Hydrogen Evolution Reaction. In Handbook of Fuel Cells; John Wiley \& Sons, Ltd: Chichester, UK, $\quad$ UK $\quad$ 2010; 1039-1044. https://doi.org/10.1002/9780470974001.f204033. 
(40) Wang, Y.; Zhang, G.; Xu, W.; Wan, P.; Lu, Z.; Li, Y.; Sun, X. A 3D Nanoporous Ni-Mo Electrocatalyst with Negligible Overpotential for Alkaline Hydrogen Evolution. ChemElectroChem 2014, 1 (7), 1138-1144. https://doi.org/10.1002/celc.201402089.

(41) Wang, M.; Wang, Z.; Yu, X.; Guo, Z. Facile One-Step Electrodeposition Preparation of Porous NiMo Film as Electrocatalyst for Hydrogen Evolution Reaction. Int. J. Hydrogen Energy 2015, 40 (5), 2173-2181. https://doi.org/10.1016/j.ijhydene.2014.12.022.

(42) Abuin, G.; Coppola, R.; Diaz, L. Ni-Mo Alloy Electrodeposited over Ni Substrate for HER on Water Electrolysis. Electrocatalysis 2019, $10 \quad$ (1), 17-28. https://doi.org/10.1007/s12678-018-0490-2.

(43) Manazoğlu, M.; Hapçı, G.; Orhan, G. Electrochemical Deposition and Characterization of Ni-Mo Alloys as Cathode for Alkaline Water Electrolysis. J. Mater. Eng. Perform. 2016, 25 (1), 130-137. https://doi.org/10.1007/s11665-015-1849-7.

(44) Geiger, S.; Kasian, O.; Ledendecker, M.; Pizzutilo, E.; Mingers, A. M.; Fu, W. T.; DiazMorales, O.; Li, Z.; Oellers, T.; Fruchter, L.; Ludwig, A.; Mayrhofer, K. J. J.; Koper, M. T. M.; Cherevko, S. The Stability Number as a Metric for Electrocatalyst Stability Benchmarking. Nat. Catal. 2018, 1 (7), 508-515. https://doi.org/10.1038/s41929-0180085-6.

(45) Wang, Z.; Zheng, Y.-R.; Chorkendorff, I.; Nørskov, J. K. Acid-Stable Oxides for Oxygen Electrocatalysis. ACS Energy Lett. 2020, $5 \quad$ (9), 2905-2908. https://doi.org/10.1021/acsenergylett.0c01625.

(46) Gong, M.; Zhou, W.; Kenney, M. J.; Kapusta, R.; Cowley, S.; Wu, Y.; Lu, B.; Lin, M.-C.; Wang, D.-Y.; Yang, J.; Hwang, B.-J.; Dai, H. Blending $\mathrm{Cr}_{2} \mathrm{O}_{3}$ into a NiO-Ni Electrocatalyst 
for Sustained Water Splitting. Angew. Chemie 2015, 127 (41), 12157-12161. https://doi.org/10.1002/ange.201504815.

(47) Hochfilzer, D.; Sørensen, J. E.; Clark, E. L.; Scott, S. B.; Chorkendorff, I.; Kibsgaard, J. The Importance of Potential Control for Accurate Studies of Electrochemical CO Reduction. ACS Energy Lett. 2021, $6 \quad$ (5), 1879-1885. https://doi.org/10.1021/acsenergylett.1c00496.

(48) Nørskov, J. K.; Bligaard, T.; Logadottir, A.; Kitchin, J. R.; Chen, J. G.; Pandelov, S.; Stimming, U. Trends in the Exchange Current for Hydrogen Evolution. J. Electrochem. Soc. 2005, 152 (3), J23. https://doi.org/10.1149/1.1856988.

\section{Table of Contents Graphic}

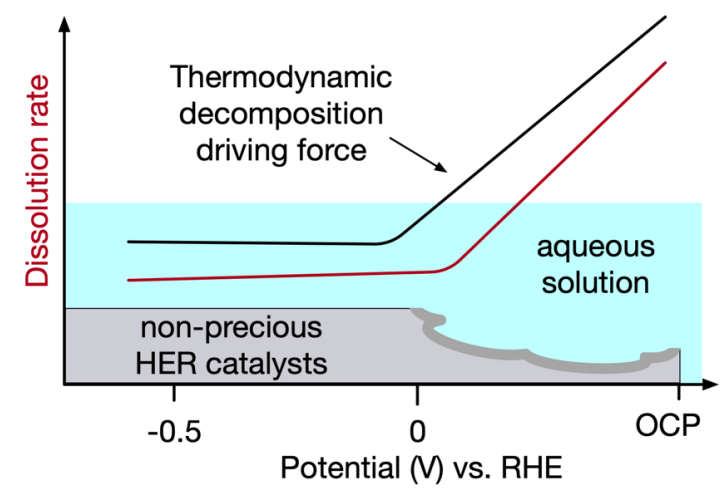

